\title{
Erratum to: Association between fat mass, lean mass, and bone loss: the Dubbo osteoporosis epidemiology study
}

\author{
S. Yang $^{1}$ • J. R. Center ${ }^{1,2}$ • J. A. Eisman ${ }^{1,2,3}$ - T. V. Nguyen ${ }^{1,4,5}$
}

Published online: 24 March 2015

(C) International Osteoporosis Foundation and National Osteoporosis Foundation 2015

\section{Erratum to: Osteoporos Int}

DOI 10.1007/s00198-014-3009-6

Owing to errors in typesetting, this article was published with an incorrect version of Fig. 1; moreover,

The online version of the original article can be found at http://dx.doi.org/ 10.1007/s00198-014-3009-6.

$\triangle$ T. V. Nguyen

t.nguyen@garvan.org.au

1 Osteoporosis and Bone Biology Research Program, Garvan Institute of Medical Research, 384 Victoria Street, Darlinghurst, NSW 2010, Australia

2 Department of Endocrinology, St Vincent's Hospital, Sydney, Australia

3 School of Medicine, The University of Notre Dame Australia, Fremantle, Australia

4 School of Public Health \& Community Medicine, Faculty of Medicine, University of New South Wales, Sydney, Australia

5 University of Technology, Sydney, Australia 
Table 3 Association between fat mass, lean mass, and relative rate of femoral neck and lumbar spine BMD change for men and women: results of multiple linear regression analysis

\begin{tabular}{|c|c|c|c|c|c|c|c|c|c|c|}
\hline \multirow[t]{3}{*}{ Adjusted variables } & \multirow{3}{*}{$\begin{array}{l}\text { Variables in } \\
\text { the model }\end{array}$} & \multirow[t]{3}{*}{ Per unit } & \multicolumn{4}{|l|}{ Men } & \multicolumn{4}{|l|}{ Women } \\
\hline & & & \multicolumn{2}{|c|}{$\begin{array}{l}\text { Femoral neck BMD } \\
\text { change (\%/year) }\end{array}$} & \multicolumn{2}{|c|}{$\begin{array}{l}\text { Lumbar spine BMD } \\
\text { change }(\% / \text { year })\end{array}$} & \multicolumn{2}{|c|}{$\begin{array}{l}\text { Femoral neck BMD } \\
\text { change (\%/year) }\end{array}$} & \multicolumn{2}{|c|}{$\begin{array}{l}\text { Lumbar spine BMD } \\
\text { change (\%/year) }\end{array}$} \\
\hline & & & Estimate (SE) & $P$ value & Estimate (SE) & $P$ value & Estimate (SE) & $P$ value & Estimate (SE) & $P$ value \\
\hline Unadjusted & Fat mass (kg) & 5 & $0.36(0.20)$ & 0.07 & $-0.11(0.19)$ & 0.57 & $-0.15(0.11)$ & 0.19 & $0.42(0.14)$ & 0.003 \\
\hline Unadjusted & Lean mass $(\mathrm{kg})$ & 5 & $0.36(0.20)$ & 0.08 & $0.28(0.19)$ & 0.14 & $-0.22(0.22)$ & 0.32 & $0.45(0.28)$ & 0.11 \\
\hline \multirow[t]{2}{*}{ Unadjusted } & Fat mass (kg) & 5 & $0.26(0.22)$ & 0.25 & $-0.28(0.21)$ & 0.17 & $-0.12(0.13)$ & 0.33 & $0.40(0.16)$ & 0.012 \\
\hline & Lean mass $(\mathrm{kg})$ & 5 & $0.25(0.23)$ & 0.28 & $0.40(0.21)$ & 0.06 & $-0.11(0.25)$ & 0.66 & $0.09(0.31)$ & 0.78 \\
\hline \multirow{2}{*}{$\begin{array}{l}\text { Adjusted for age, height, } \\
\text { history of prior fracture, } \\
\text { smoking, osteoarthritis } \\
\text { and physical activity }\end{array}$} & Fat mass (kg) & 5 & $0.22(0.22)$ & 0.33 & $-0.26(0.21)$ & 0.21 & $-0.13(0.13)$ & 0.34 & $0.36(0.17)$ & 0.03 \\
\hline & Lean mass $(\mathrm{kg})$ & 5 & $0.20(0.30)$ & 0.51 & $0.47(0.28)$ & 0.09 & $-0.17(0.29)$ & 0.56 & $0.13(0.37)$ & 0.73 \\
\hline
\end{tabular}

Statistical significance (at $5 \%$ level) are shown in italics 